\title{
Time-changed Lévy processes and option pricing: a critical comment ${ }^{*}$
}

\author{
Hasan Fallahgoul ${ }^{\dagger}$ \\ Kihun $\mathrm{Nam}^{\ddagger}$ \\ Monash University \\ Monash University
}

July 3, 2019

\begin{abstract}
Carr and $\mathrm{Wu}$ (2004), henceforth CW, developed a framework that encompasses almost all of the continuous-time models proposed in the option pricing literature. Their framework hinges on the stopping time property of the time changes. By analyzing the measurability of the time changes with respect to the underlying filtration, we show that all models CW proposed for the time changes fail to satisfy this assumption.
\end{abstract}

Carr and Wu (2004), henceforth CW, developed a time-changed Lévy (TCL) framework that encompasses almost all of the continuous-time models proposed in the option

${ }^{*}$ We would like to thank Julien Hugonnier for motivating this note and for fruitful discussions. We also thank Samuel Cohen, Yan Dolinsky, Gregoire Loeper, Loriano Mancini, Stoyan Stoyanov, Farshid Vahid, and seminar participants at QMF 2018 and the 62nd Annual Meeting of the Australian Mathematical Society for comments. The Centre for Quantitative Finance and Investment Strategies has been supported by BNP Paribas.

${ }^{\dagger}$ Hasan A. Fallahgoul, Monash University, School of Mathematics and Centre for Quantitative Finance and Investment Strategies, 9 Rainforest Walk, 3800 Victoria, Australia. E-mail: hasan.fallahgoul@monash.edu.

${ }^{\ddagger}$ Kihun Nam, Monash University, School of Mathematics and Centre for Quantitative Finance and Investment Strategies, 9 Rainforest Walk, 3800 Victoria, Australia. E-mail: kihun.nam@monash.edu. 
pricing literature by allowing a correlation between the Lévy process and stochastic time. The whole paper is based on their closed-form expression of the characteristic function for the TCL process, ${ }^{1}$ which hinges on the stopping time property for the stochastic time. ${ }^{2}$ Though their closed-form expression for the characteristic function is correct when the stochastic time is a collection of stopping times, none of the specifications proposed by $\mathrm{CW}$ for the time changes satisfies this condition, as the stochastic time is given only as a process adapted to the underlying filtration.

It is not obvious, if not impossible, to construct the stochastic time $T=\left(T_{t}\right)_{t \geq 0}$ such that (i) it is correlated to the Lévy process; (ii) the time change $T_{t}$ is a stopping time for each $t$; (iii) the Laplace transforms of $T_{t} \mathrm{~s}$ with respect to the leverage-neutral complex measure can be expressed in closed form; and (iv) it captures empirical regularities such as stochastic volatility and volatility clustering. In fact, it is more natural to assume that the stochastic time is an adapted process. In the CW's framework, the asset log-return is modeled by $X_{T}$, a Lévy process $X$ that runs on a continuous stochastic time $T$. By the Lévy-Itô decomposition theorem, the continuous part of the Lévy process $X$ is a Brownian motion with drift. Since $T_{t}=\frac{t}{\mathbb{E} Q_{t}} Q_{t}$ where $Q$ is the continuous part of the quadratic variation of the asset $\log$-return $X_{T}$, we know $T$ can be observed from the market. Therefore, the adaptedness assumption on $T$, which was used in the specifications in CW and literature, is appropriate in the financial context.

One possibility to apply the CW's framework to their specifications is to change the

\footnotetext{
${ }^{1}$ The closed-form expression for the characteristic function in $\mathrm{CW}$ is given by the Laplace transform of stochastic time $T$ evaluated at the characteristic exponent of $X$ under the leverage-neutral measure, which is a complex measure.

${ }^{2}$ For a given filtration $\left(\mathcal{F}_{s}\right)_{s \geq 0}$, we say a stochastic time $T=\left(T_{t}\right)_{t \geq 0}$ has the stopping time property if $\left\{T_{t} \leq s\right\} \in \mathcal{F}_{s}$ for all $s, t \geq 0$.
} 
filtration so that the $T_{t}$ s become stopping times and $X$ remains Lévy. If the Lévy process $X$ and the stochastic time $T$ are independent, then the filtration change poses no problem. However, when $X$ and $T$ are dependent, the filtration change that makes $T_{t} \mathrm{~s}$ stopping times will affect the semimartingale property of $X$, and this will lead us to an arbitrage opportunity. Let us clarify with an example. For a positive constant $\rho$ and two independent Brownian motions $W$ and $B$, consider the case where $X=\rho W+\sqrt{1-\rho^{2}} B$ and $T_{t}=\int_{0}^{t} \exp \left(W_{s}-s^{2} / 2\right) d s$. The minimal filtration that makes $T_{t}$ s stopping times and $X$ adapted is $\mathbb{G}:=\left(\mathcal{F}_{C_{t}}^{W} \vee \mathcal{F}_{t}^{X}\right)_{t \geq 0}$, where $C_{t}$ is the first $t$-level crossing time of $T$ (see Proposition A.1). This implies that under $G$, one can determine the value of $C_{t}$ and the path of $W$ from time 0 to $C_{t}$ at time $t$. If $C_{t}>t$ happens at $t, X$ from time $t$ to $C_{t}$ can be decomposed into $\mathbb{G}$-martingale $\sqrt{1-\rho^{2}} B$ and deterministic path $\rho W$ with infinite first-order variation. Therefore, $X$ is no longer a semimartingale under $G$ and it has an arbitrage opportunity. In particular, if we set $\rho=1$, then we can set an arbitrage strategy as follows: Since $C_{t}$ is adapted to $G$, we can determine whether $C_{t}>t+1$ or not at time $t$. At time $t$, if $C_{t}>t+1$, then we can find the path of $X$ from $t$ to $C_{t}$ and the asset price at time $t+1$. If the asset price at $t+1$ is higher than the asset price at $t$, you buy the asset at time $t$, otherwise, you short the asset.

The above argument is based on Proposition A.1 which tells us the following: Under the enlarged filtration that makes $T$ being stopping time and adapted, we are able to foretell, not forecast, the future of the business activity rate $v=\frac{d T}{d t}$ as well as the past of it. Let us provide an example from CW. Let the business activity rate $v$ be a Cox-IngersollRoss (CIR) process, which is an affine activity rate model (see Section 4.2.1 of CW). The left panel of Figure 1 provides a simulation of the CIR process with parameters from Table 1 in 
Fallahgoul et al. (2019), while the right panel shows the integral of the simulated business activity rate that represents $T$. Since the calendar time $t$ is replaced by the time change $T_{t}$, one needs to impose the condition that the unconditional expectation of $T_{t}$ is $t$. This implies that a realized path of $T$ always oscillates around the line $y=t$, as the right panel of Figure 1 shows. Since the business activity rate $v$ is adapted, its integrated process $T$ is adapted as well. If $T_{t}$ is a stopping time for each $t$, then based on Proposition 1 , we know the path of $T$ until it hits the level of $t$ at time $t$. Assume $T_{t}>t$ at time $t=1.2$ as in the right panel of Figure 1 . Then, at time $t=1.2$, we know the whole path until $S$ using the adaptedness of $T$. Since the stopping time property of $T_{t}$ for all $t$ requires knowledge of the path only until $R$, there is no problem in this respect. Alternatively, assume that $T_{t}<t$ at time $t=0.6$, as in the right panel of Figure 1 . Then, the stopping time property of $T_{t}$ for each $t$ implies that we know the whole path of $T$ until $Q$ instead of $P$ at time $t=0.6$. This is impossible under the natural filtration that defines the CIR process. Therefore, we cannot use the CIR process for model stopping times in CW's framework.

$$
\text { Insert Figure } 1 \text { about here }
$$

In sum, unfortunately, there are several ways to show that none of the specifications proposed in $\mathrm{CW}$ for the stochastic time satisfies their assumptions. Perhaps more importantly, the construction of stochastic processes that satisfy their assumptions and are empirically friendly, meaning one can obtain their characteristic functions from CW's results, is challenging and still an open topic for further investigation.

Fallahgoul and Nam (2019) extend CW's result to a case in which the stochastic time T is just an adapted process. The model becomes flexible enough to incorporate virtually all 
models proposed in the option pricing literature. In this case, all specified option pricing models in Huang and $\mathrm{Wu}(2005)$ are nested in our setting.

\section{References}

Carr, P., and L. Wu. 2004. Time-changed Lévy processes and option pricing. Journal of Financial Economics 71:113-141.

Fallahgoul, H., J. Hugonnier, and L. Mancini. 2019. Time changes, Lévy jumps and asset returns. Monash CQFIS working paper .

Fallahgoul, H., and K. Nam. 2019. Correlated time-changed Lévy processes. Monash CQFIS working paper.

Huang, J. Z., and L. Wu. 2005. Specification analysis of option pricing models based on time-changed Lévy processes. Journal of Finance 59:1405-1439. 

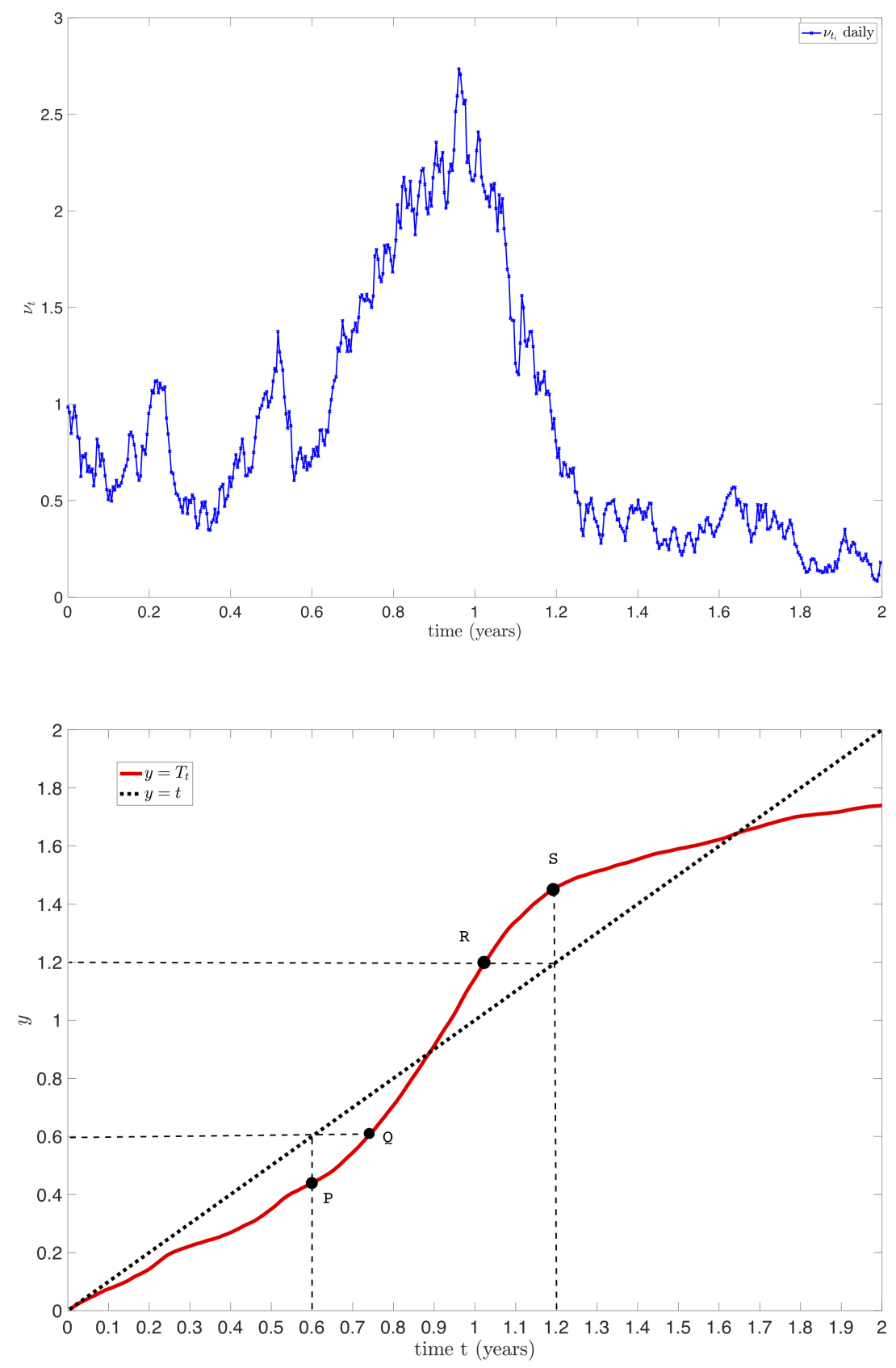

Figure 1: Simulated CIR process.

(top) Two years simulated Cox-Ingersoll-Ross (CIR) process, i.e., $d v_{t}=\kappa\left(\theta-v_{t}\right) d t+$ $\sigma \sqrt{v_{t}} d W_{t}$, where $W_{t}$ is a Brownian motion, parameter $\kappa$ corresponds to the rate of mean reversion, $\theta$ is the long-runs mean, and $\sigma$ capturs volatility. (down) Integrated CIR process. $T_{t}=\int_{0}^{t} v_{s} d s$. 


\section{A Adaptedness and the stopping time property}

Let $\mathbb{F}=\left(\mathcal{F}_{t}\right)_{t \geq 0}$ be the underlying filtration in $\mathrm{CW} .^{3} \mathrm{In}$ their paper, the stochastic time $T$ is an absolutely continuous process and satisfies two assumptions: $T_{t}$ is a $\mathbb{F}$-stopping time for each $t$, and for $s \leq t, T_{s}$ is $\mathcal{F}_{t}$-measurable. The first assumption is used to derive the characteristic function or generalized Fourier transform of the TCL process (see Lemma 1 of $C W$ ), while the second assumption is used to specify the business activity rate $v_{t}=\frac{d T_{t}}{d t}$ in Section 4.2 of CW.

These two assumptions imply that, at time $t$, we can determine when the stochastic time $T$ hits the $t$-level, as well as the path of $T$ until it hits the $t$-level.

Proposition A.1. Let $\left(T_{t}\right)_{t \geq 0}$ be an increasing continuous process. Assume that $\mathbb{F}:=\left(\mathcal{F}_{t}\right)_{t \geq 0}$ is the filtration such that $T$ is adapted and $T_{t}$ is a $\mathbb{F}$-stopping time for each $t \geq 0$. Let $C_{s}:=$ $\inf \left\{t>0: T_{t}>s\right\}$ be the first s-level crossing time of $T$. Then, for all $t \geq 0, C_{t}$ is $\mathcal{F}_{t}^{T}$ measurable. Moreover,

$$
\mathcal{F}_{C_{t}}^{T} \subset \mathcal{F}_{t}^{T} \subset \mathcal{F}_{t}
$$

where $\left(\mathcal{F}_{t}^{T}\right)_{t \geq 0}$ is the filtration generated by $T$.

Proof. Note that $C_{t}$ is $\mathcal{F}_{t}^{T}$-measurable for every $t \geq 0$ because

$$
C_{t}^{-1}([s, \infty))=\left\{C_{t} \geq s\right\}=\left\{T_{s} \leq t\right\} \in \mathcal{F}_{t}^{T}
$$

for any $s \in \mathbb{R}$ and $\{[s, \infty): s \in \mathbb{R}\}$ generates Borel sigma algebra in $\mathbb{R}$. Therefore, $\mathcal{F}_{t}^{C} \subset$ $\mathcal{F}_{t}^{T}$, where $\left(\mathcal{F}_{t}^{C}\right)_{t \geq 0}$ is the filtration generated by $C$. We now prove $\mathcal{F}_{C_{t}}^{T} \subset \mathcal{F}_{t}^{C}$.

Since $T$ is a continuous increasing function, $T_{s}=\inf \left\{u \geq 0: C_{u}>s\right\}$. Therefore, $T_{s}$ is the first $s$-level crossing time of $C$. This implies that $\mathcal{F}_{s}^{T} \subset \mathcal{F}_{T_{s}}^{C}$. Now, if we let $s=C_{t}$ and use the relationship $T_{C_{t}}=t$, we have

$$
\mathcal{F}_{C_{t}}^{T} \subset \mathcal{F}_{t}^{C}
$$

which completes the proof.

\footnotetext{
${ }^{3}$ Throughout $\mathrm{CW}$, the filtration they refer to when they mention martingale, stopping time, and the Lévy process is not clear. To make Lemma 1 of $\mathrm{CW}$ correct, we need to assume that $T_{t}$ are stopping times with respect to filtration $\mathbb{F}$ such that $X$ is the Lévy process, while $C W$ assume that $T$ is adapted to $\mathbb{F}$ in Section 4.3.1. For consistency, we assume that $\mathrm{CW}$ used the same filtration throughout their paper.
} 